\title{
THE EFFECTS OF A COMPLEX TROPHIC STRUCTURE OF MAMMALIAN HOST SPECIES ON THE ECOLOGY ON EMERGING INFECTIOUS DISEASES
}

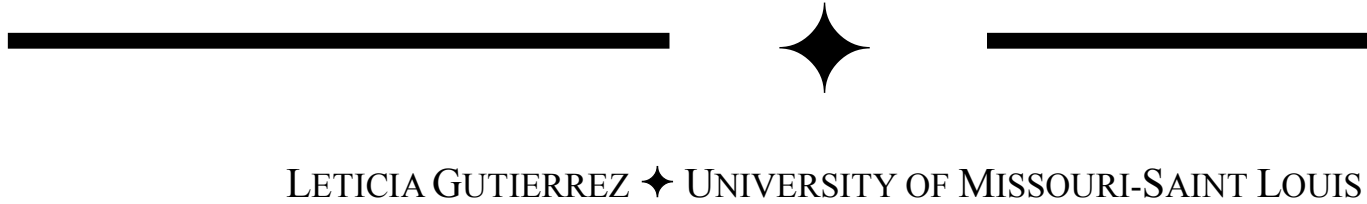

\section{$\downarrow \quad$ INTRODUCTION}

The benefit of a complex, intact community for maintaining ecosystem health in the face of emerging infectious disease risk has not been deeply explored. The diversity and distribution of haemoparasites in potential host mammal fauna are virtually unknown, and many diseases endemic to North America are not well understood in terms of transmission factors, prevalence, and contagion. Many of these tick and rodent borne pathogens nowadays are considered to be potential emerging infectious diseases that could spread to adjacent areas and new hosts, including humans, with climate change, landuse shift, and the expansion of distributions of the natural vectors of such haemoparasites. This study examines the effect of mammal community complexity in maintaining ecosystem health with respect to rodent/tick-borne diseases which have a high value for human public health as zoonotic diseases as well as for the unknown natural history of the mammalian community network.

At this point, a crucial step towards understanding the influence of parasites on mammal populations and emerging infectious disease (EID) risk is simply to describe the distribution of parasites within the entire mammal community and characterize the interconnections between pathogens, ectoparasite vectors, hosts species and land-use.

The work described in this brief report will provide the basis for the second and third chapters of the dissertation of doctoral student Leticia Gutiérrez, which will be an integrative study of mammal species that are connected through trophic and parasitic networks. The Greater Yellowstone Ecosystem
(GYE), which is the last example of a well-conserved temperate ecosystem in the northern hemisphere, will provide the setting for a first step towards characterizing the haemoparasite community in a mammal community with a full complement of top predators. This work will help to clarify the influence of carnivores and large herbivores, and other less known interactions, on parasite infection dynamics in local rodent communities. One direct benefit will be recommendations for decreasing the risk of human EIDs transmitted by ticks. Thus, the results of this study might reduce the negative perception of the local community of ranchers toward carnivores. The present report covers resources required to characterize bloodborne pathogens in a large sample of rodents captured in paired locations around the GYE contrasting high and low human disturbance involving ranching and recreation.

\section{$\uparrow \quad$ HYPOTHESIS}

Land use intensity is directly related to complexity of communities of mammalian host species which, in turn, influences the overall health of the ecosystem by moderating disease risk and pathogen transmission.

\section{$\uparrow \quad$ PREDICTION}

Areas protected from human disturbance will sustain a higher diversity of mesocarnivores, large carnivores, and, in general, large vertebrates, which will negatively affect the abundance of rodent species. As a consequence, tick-borne diseases will be less prevalent in undisturbed areas compared to areas with high levels of human disturbance. 


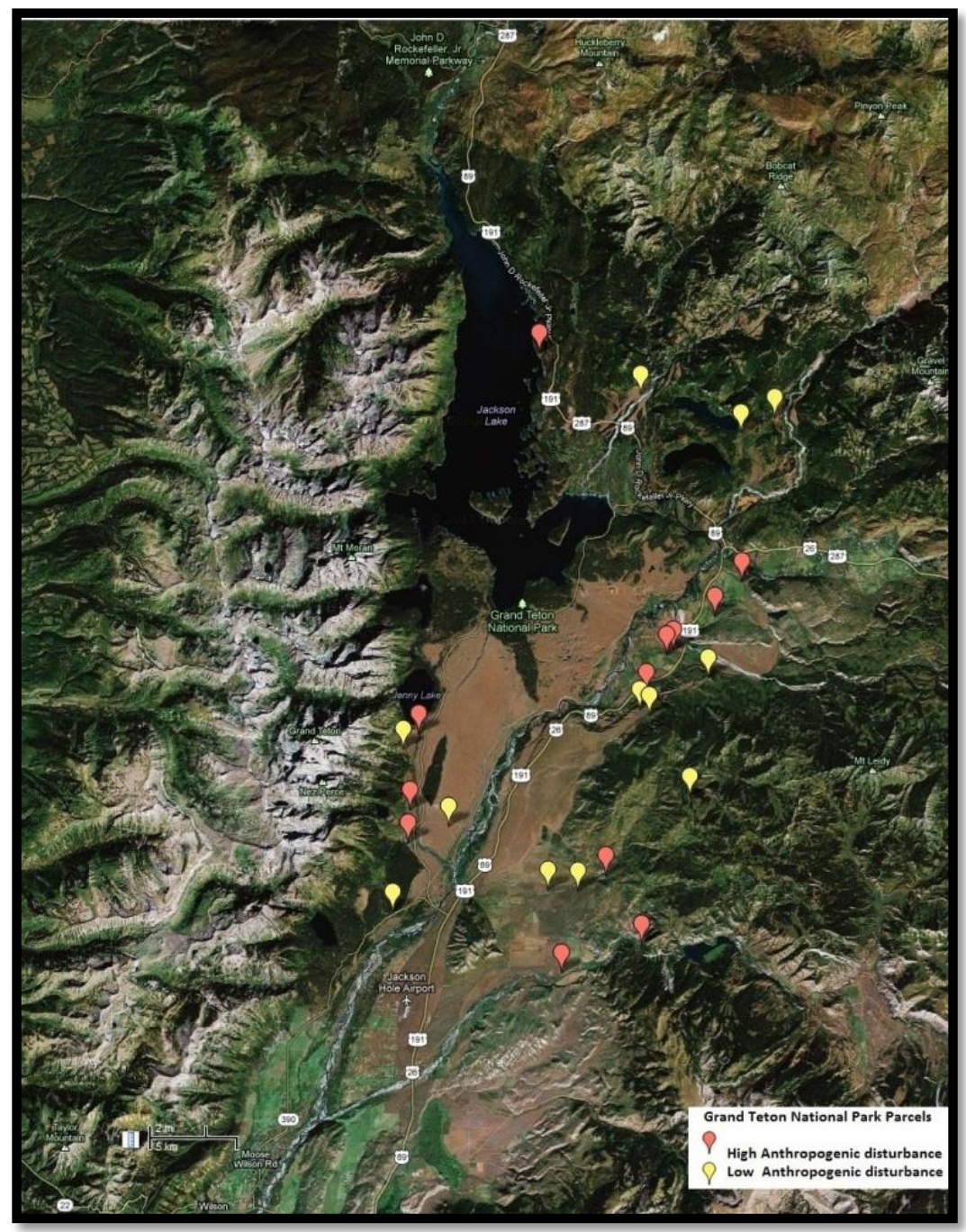

Figure 1. Grand Teton National Park rodent trapping showing high showing areas of high and low anthopogenic disturbance.

\section{$\uparrow$ OBJECTIVES}

1) Determine the variety, abundance, and distribution of tick-borne pathogens of rodents in the GYE.

2) Determine the patterns of disease prevalence and interactions in the host-parasite-vector network.

3) Determine the direct and indirect effects of maintaining a complex trophic structure in the local mammalian fauna on disease risk indicators and overall ecosystem conservation, particularly with respect to habitat alteration and patterns of land use.

\section{$\uparrow \quad$ STUDY AREA}

The field study is located in the GYE, in Grand Teton National Park $\left(43^{\circ} 44^{\prime} 0^{\prime \prime} \mathrm{N}\right.$ $\left.110^{\circ} 48^{`} 12^{\prime \prime} \mathrm{W}\right)$ (Figure 1).

\section{$\uparrow \quad$ MAMMALIAN TRAPPING AND BLOOD SAMPLE COLLECTION}

During summer 2012, twenty-four parcels were chosen for this purpose, divided by anthropogenic disturbance and land-use/vegetation cover: 4 grazing plots, 4 horse/cattle ranches, and 4 settlements as part of the highly disturbed areas lacking large mammals and 4 pristine meadows, 4 pristine sagebrush plots, and 4 pine forest plots as part of the undisturbed areas where a complex trophic structure in the mammalian community is expected to occur (Figure 1). During the 2012 field season at GTNP, 1,208 rodent samples (Figure 2) were added to the data source. This effort was achieved by doctoral student Leticia Gutiérrez as project leader, and three additional undergraduate field assistants. 


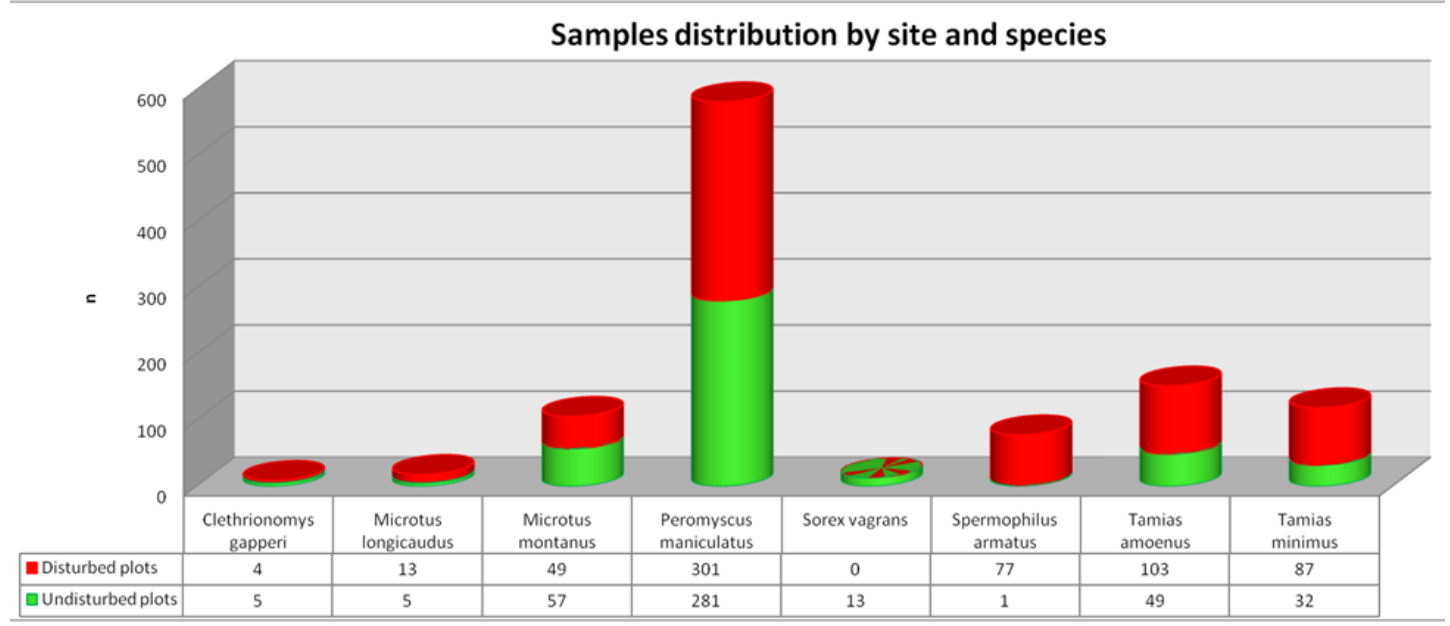

Figure 2. Rodent blood samples collected within Grand Teton National Park during summer 2012, $\mathrm{n}=1,208$ :

Teams of two people each sampled two sites each five days, over a period of eleven weeks, making it possible to access twenty-four new plots during the three month summer at GYE. Oats, sunflower seeds and dry raisins were used as bait. Traps were baited at sunset and checked the following morning at sunrise. Traps were closed during the day, and at night they were provided cotton pads to avoid hypothermia. Trapping was done during four consecutive nights. All traps were numbered; all sites were marked using colorful flagging and were GPS georeferenced. All individuals captured were identified with numbered ear tags, weighed, and sexed.

From each Isoflurane anesthetized mammal, blood was collected in a $40-\mu 1$ capillary tube from the sub-mandibular vein or saphenous vein. Part of the sample was preserved in Elute FTA cards/ Longmire's lysis buffer and part was used to make a smear. Serum samples also were collected. This blood collection protocol was chosen since this method is the least invasive among others described for small size mammals. Each trapped mammal was checked through direct visual inspection, and ectoparasites were collected with anatomical fine tweezers. Ectoparasites (ticks and flies) were kept in Eppendorf vials for $24 \mathrm{hrs}$ in order to let the blood meals with the host DNA be thoroughly digested. The parasites were preserved in $1 \mathrm{ml} 70 \%$ ethanol, frozen and subsequently lyophilized. Hence, it will be possible to preserve the ectoparasite DNA until it can be processed in the PI's lab.

Parasite DNA isolated from mammalian blood samples is subjected first to a reverse line blot (RLB, see Table 1) assay to identify, in a cost-effective manner, bacterial and piroplasma haemoparasites. 5'- end amino-link species-specific probes are blotted in lines using a Miniblotter 45 (Immunetic, Cambridge, MA). Results are detected using a chemiluminescent substrate in autorad film. Being able to screen 43 samples against 42 different probes at one time makes this an excellent tool for the first stage of the molecular analysis. DNA of the detected pathogens will be amplified by multiplex PCR using biotin-labeled general primers for eubacteria and piroplasma. Oligonucleotide sequences of bacterial and piroplasma probes used in RLB assay are: Anaplasma phagocytophilum, Arsenophonus sp., Borrelia. afzelii, $B$. burgdorferi sensu lato, B. burgdorferi sensu stricto, $B$. burgdorferi sensu lato $16 \mathrm{~S}, B$. lonestari, $B$. valaisiana, B. garinii, Ehrlichia canis/ovis/muris, E. ewingii, E. chaffeensis, Francisella endosymbiont of Dermocentor variabilis, F. philomiragia, F. tularensis subsp. tularensis, F. tularensis $+F$. philomiragia, Rickettsia amblyommii, R. amblyommii + Rickettsia sp., Rickettsia endosymbiont of Dermocentor variabilis, $R$. rickettsii, Babesia bigemina, B. bovis, $B$. caballi, $B$. canis 1, B. canis 2, $B$. catch-all 1, Babesia catch-all 2, B. divergens, $B$. felis, B. microti $1, B$. microti 2, B. ovis, B.rossi, B. vogeli, Hepatozoon catch-all, Theileria annae, T. annulata, T. buffeli, Theileria catch-all, $T$. equi, $T$. parva and Theileria/Babesia catch-all.

In addition, PCR will be carried out using taxon-specific primers. In the case of positive infections, 18S rDNA, 16S rDNA, mitochondrial cytochrome $\mathrm{b}$, or other specific genetic markers will be sequenced for parasite identification. According to objectives established in this project, we will identify Plasmodium, Rickettsia, Babesia, Borrelia, Ehrlichia, Hepatozoon, Anaplasma, Theileria, and Francisella haemoparasites. DNA sequencing will be done by the 
DNA Service at the Genomics Core Facility at PennState.

It is important to point out that this is the first survey of parasites to detect $B$. burgdorferi, the agent of Lyme disease, outside of the Northeast, Upper Midwest and California distribution range described for this highly pathogenic zoonotic disease. This novel finding can contribute to the study of climate change (e.g. expansion in tick vector, host shift and disease distribution ranges) and to understanding the protective role of rich trophic networks.

This preliminary data shows strong evidence of specific rodent borne diseases associated with disturbed areas ( $\mathrm{p}$-value $=0.009$ ). Individual characteristics such as species, sex and age seems to be important regarding haemoparasite prevalence. For instance, Borrelia borgdorferi prevalence appears to be influenced by the host species $(p$-value $=0.01)$ and also by the interaction of sex and age of the individuals $(p$-value $=0.004)$. In this particular case, all the infected individuals with $B$. burgdorferi were trapped in disturbed areas. Surprisingly, $45 \%$ of them belong to the species Tamias minimus (least chipmunk) and just $22 \%$ to the species Peromyscus maniculatus (white-footed mouse). In addition, $22 \%$ of the individuals correspond to female-adults, $56 \%$ to maleadults and $22 \%$ to male-juveniles. These novel findings might unravel new host species as more competent disease reservoirs.

More extensive genetic studies will be focused on the Lyme disease population distribution and expansion. In addition to RLB assay, DNA extracts from hosts and vectors will be screened by PCR with genus-specific flaB primers. PCR amplicons will be directly sequenced. Sequence diversity and genetic diversity will be assessed. Sequence variants at $f l a B$ will delimit Borrelia species and subtypes among species. Published pathogen sequences in GenBank will be used as alignment template; MEGA 5 will be used to determine genetic distances. Maximum likelihood phylogenetic tree building will be made for each pathogen species found using Geneious. Phylogenetic tree will be visualized with FigTree software.
In order to understand the spread of Lyme disease through the continent it is necessary to explore the population structure of Borrelia burgdorferi correlated with the spatial distribution of sequence types. Multilocus sequence analysis of eight chromosomal housekeeping genes (clpA, clpX, nifS, pepX, pyrG, recG, rplB and uvrA) of the spirochete strains will elucidate the phylogenetic relationship of the newly described B. burgdorferi isolates. The MLST data will be analyzed regarding spatial distribution of sequence types (STs), correspondence between spatial distribution of STs and geographic location through permutation test and Bayesian analysis of the population structure will assess the relatedness of the B. burgdorferi strains.

Data analysis will be based upon haemoparasite prevalence among rodent species. Cluster analysis will be used to assess the geography of disease prevalence. Then, univariate analysis will be performed to assess disease prevalence differences among clusters and land use. Finally, logistic regression will be used to take into account the different host and environmental variables, and their interactions, which might influence inter- and intraspecific disease prevalence.

This project addresses a major gap in current knowledge and understanding of EID dynamic patterns and risk factors through an empirical study of the semi-pristine area of the GYE. This comprehensive in situ study integrates a diverse array of disciplines such as public health, disease ecology, epidemiology, and conservation, which will lead to increased understanding of the role of a complex trophic structure in disease dynamics.

Exploring how preserving high species diversity of a mammalian community, along with a strong complexity in all its trophic levels, can have an intrinsic measurable value to human health. This will be used to address conservation policies and management. EID risk models will directly benefit the human populations, bringing together new land-use management that will benefit humans as well as protect wildlife. 